\title{
STUDY OF IOVE AND RAYLEIGH WAVES FROM EARTHQUAKES WITH FAULT PLANE SOLUTIONS OR WITH KNOWN FAULTING. PART 1. A PHASE DIFFERENCE METHOD BASED ON A NEW MODEL OF EARTHQUAKE SOURCE
}

\author{
By KeIITI AKI
}

\begin{abstract}
For the purpose of examining the basic assumptions underlying the surface wave method of earthquake mechanism study, we investigated Love and Rayleigh waves from earthquakes with known faulting and/or fault plane solutions obtained from initial motion studies. In order to eliminate the effect of the source time function and finiteness of the fault and to concentrate on the nature of the earthquake force system and its space parameters, we are primarily concerned with the phase differences between Love and Rayleigh waves and their amplitude ratios.

We studied about 30 earthquakes which occurred in the Mediterranean region, California, and Japan. The results are given in Part 2, and the method used is described in the present paper. The theoretical phase and amplitude of Love and Rayleigh waves were computed on the basis of observed faulting or fault plane solution under various hypotheses about the equivalent force system. Then, we obtained from the record, the Fourier phase difference of Love and Rayleigh waves, corrected it for propagation in a layered earth and compared it with the corresponding theoretical value.

In computing the theoretical values, we assumed a homogeneous half space for Rayleigh waves. For Love waves, the layered structure of the earth was taken into account in an approximate way. We have constructed a table of the theoretical values for all possible parameters of fault system and also for various focal depths. A part of the table is given in a concise form in Part 3.

The measurement of the phase difference between Love and Rayleigh waves was made by two methods. One is the stationary phase analysis, first applied to seismograms by Brune, Nafe and Oliver (1960), and the other is a filtering-correlation method. The latter method is appropriate for those records where the waves are less dispersed and noise is a factor.

It was found that the single couple hypothesis fails to explain the observations on surface waves, and must be modified in some way. A modified single couple hypothesis is proposed which appears to explain the observations generally better than the double couple hypothesis as will be shown in Part 2.
\end{abstract}

\section{INTRODUCTION}

The purpose of the present paper is to examine the validity of the basic assumptions underlying the surface wave method of earthquake mechanism study by investigating waves from earthquakes with known faulting and/or fault plane solutions obtained from initial motion studies. We will pose the following questions: Is the fault formed at the time of an earthquake responsible for seismic radiation? Is the source essentially the same for long period surface waves as for short period body waves? Which type of simple force system best represents the earthquake source, single couple, double couple or some other mechanism? Affirmative answers to the first two questions will be given in Part 2. As to the force system, we shall propose a new model, which may be applicable to earthquakes with shallow focal depths and has an intermediate character between the single couple and the double couple. 
The determination of earthquake mechanism from surface waves involves many difficult problems. The most serious problem is the uncertainty in the phase velocity data used in the correction of the observed phase spectra of these waves for propagation. Aki (1962a) discussed the error due to this uncertainty for several practical cases and concluded that the phase velocity data presently available are not accurate enough to get a precision in the source phase better than $\pi / 4$.

Another problem is the interpretation of the observed source phase and amplitude of these waves in terms of the source parameter of an earthquake. Even if we take a simple force system such as a single couple or a double couple, as an equivalent source, there are many additional parameters which we must take into consideration. These are the dip direction and dip angle of the fault plane, the motion direction in the fault plane, the time function of the applied force, the finiteness of the fault, and the depth of the fault.

The accumulation of good quality data on these waves through a long period seismograph network such as the world-wide standard station network will eventually solve the problem of the phase velocity data and also may give us sufficient observations for obtaining a unique solution for the source mechanism from surface waves alone.

In the meantime, however, we may take an alternative approach to the problem. In the present paper, we shall not deal with the absolute value of the phase spectra of Love and Rayleigh waves but the difference between the two spectra. Since the variation in crustal structure affects the phase velocities of Love and Rayleigh waves generally in a similar manner, the correction required for obtaining the source phase difference between the two waves will be less dependent on crustal structure variation than for individual waves.

Further, we can greatly simplify our problem of interpretation by taking the difference between the phase spectra of Love and Rayleigh waves. First, the effect of the source time function is cancelled out in the difference. Secondly, the effect of finiteness of the fault, such as postulated by Ben-Menahem (1961) will approximately be cancelled. Thus, we can concentrate on the basic assumption of the force system and its space parameters.

This method is comparable to the method based on the polarization of $S$ waves (Stauder, 1962), because we study the relation between radial and transverse motion as evidenced by the form of Rayleigh and Love waves. There is, however, a significant difference between the two methods. $S$ wave motion at long distance is polarized linearly if the propagation effect is corrected for, and there is no phase difference between $S V$ and $S H$ waves due to the source effect. On the other hand, if we synthesize the radial component of Rayleigh waves and the transverse component of Love waves after correcting for propagation, we get in general an elliptic motion with arbitrary axes rather than a linear motion. Thus, we have one more observable parameter pertinent to the source in our method than in the $S$-wave polarization method.

In the present paper, we shall first describe the general method and various practical techniques used in the computation of the phase difference from the actual record. Secondly, we shall discuss the propagation correction and describe the theoretical phase and amplitude of Love and Rayleigh waves for an arbitrary fault 
system under the assumption of a single couple, double couple and our new model. Then, in Part 2 we shall apply our method to earthquakes in various regions of the earth.

\section{Method}

Let the radial component of displacement of Rayleigh waves be $R(t)$ and the transverse component of Love waves be $L(t)$. Outward motion from the source is taken positive in the former, and the left hand motion as seen from the source is taken positive in the latter. We may express them as

$$
L(t)=\frac{1}{\pi} \int_{0}^{\infty}\left|A_{L}(\omega)\right| \cos \left(\omega t+\phi_{L P}+\phi_{I}+\phi_{L}\right) d \omega
$$

and

$$
R(t)=\frac{1}{\pi} \int_{0}^{\infty}\left|A_{R}(\omega)\right| \cos \left(\omega t+\phi_{R P}+\phi_{T}+\phi_{R}\right) d \omega
$$

Here, $\phi_{L P}$ and $\phi_{R P}$ are the phase shifts due to propagation, $\phi_{I}$ is the instrumental phase shift and $\phi_{L}$ and $\phi_{R}$ are the phase shifts produced at the source.

From actual records, we can obtain the Fourier amplitudes $\left|A_{R}\right|$ and $\left|A_{L}\right|$ and the phases $\phi_{L}{ }^{\prime}$ and $\phi_{R}{ }^{\prime}$ defined in the following formulas,

$$
L(t)=\frac{1}{\pi} \int_{0}^{\infty}\left|A_{L}(\omega)\right| \cos \left(\omega t+\phi_{L}^{\prime}\right) d \omega
$$

and

$$
R(t)=\frac{1}{\pi} \int_{0}^{\infty}\left|A_{R}(\omega)\right| \cos \left(\omega t+\phi_{R}{ }^{\prime}\right) d \omega
$$

Then, the source phase difference between Love and Rayleigh waves is obtained by the formula,

$$
\phi_{R}-\phi_{L}=\phi_{R}{ }^{\prime}-\phi_{L}{ }^{\prime}-\left(\phi_{R P}-\phi_{L P}\right)
$$

Since the polar phase shift term (Brune et al., 1961) is common for both waves, we can write

$$
\phi_{R P}-\phi_{L P}=\left(\frac{1}{C_{L}}-\frac{1}{C_{R}}\right) \omega \Delta
$$

where $C_{L}$ and $C_{R}$ are the phase velocities of the Love and Rayleigh waves respectively, $\Delta$ is the epicentral distance, and $\omega$ is the angular frequency.

In order to obtain the Fourier phase difference $\phi_{R}{ }^{\prime}-\phi_{L}{ }^{\prime}$, we may apply one of the following three techniques depending on the nature of the records: (1) Fourier 
analysis, (2) stationary phase analysis, or (3) band-pass-filtering and correlation analysis. The Fourier analysis method is well known and need not be explained here. The stationary phase analysis was first applied to seismograms, by Brune, Nafe and Oliver (1960). This is a very quick and convenient method fitted to dispersed wave trains. We shall describe how this technique is used in our analysis.

\section{Stationary Phase Analysis}

For large $t$, the integral of the form of equation 2 may be evaluated by the method of stationary phase and shown to be approximately

$$
L(t) \sim \frac{\left|A\left(\omega_{0}\right)\right|}{\sqrt{2 \pi\left|\frac{d^{2} \phi}{d \omega^{2}}\right|_{0}}} \cos \left(\omega_{0} t+\phi\left(\omega_{0}\right) \pm \frac{\pi}{4}\right)
$$

according as $d^{2} \phi / d \omega^{2} \gtrless 0$. Here, $\omega_{0}$ is given by the stationary phase condition

$$
t=-\left(\frac{d \phi}{d \omega}\right)_{\omega=\omega_{0}}
$$

Then, the time $t_{p N}$ at which the $N$ th peak of the wave appears is given by

$$
\omega_{0} t_{p N}+\phi\left(\omega_{0}\right) \pm \frac{\pi}{4}=2 N_{\pi}
$$

and the time $t_{t N}$ at which the $N$ th trough appears is given by

$$
\omega_{0} t_{t N}+\phi\left(\omega_{0}\right) \pm \frac{\pi}{4}=(2 N+1)_{\pi}
$$

Here, the $\omega_{0}$ 's are different for different peaks and troughs and are given by equation 6.

The practical steps in obtaining the source phase difference between Love and Rayleigh waves by this technique are illustrated in table 1 . Column 1 shows the times of peaks and troughs of Love waves from a Mediterranean shock recorded at Pasadena. The period corresponding to each peak and trough is obtained from the slope of the curve of wave number plotted against arrival time, and is shown in Column 2.

Column 3 shows the products of period and wave number. They are subtracted from the corresponding arrival times and the results are shown in Column 4 . The numbers in this column may be expressed as

$$
-\frac{\phi_{L}{ }^{\prime}\left(\omega_{0}\right) \pm \frac{\pi}{4}}{\omega_{0}}=t_{p N}-\frac{2 N \pi}{\omega_{0}}
$$


or

$$
-\frac{\phi_{L}{ }^{\prime}\left(\omega_{0}\right) \pm \frac{\pi}{4}}{\omega_{0}}=t_{t N}-\frac{(2 N+1) \pi}{\omega_{0}}
$$

TABLE 1

An Illustration of the Determination of Source Phase Difference Between love and Rayleigh Waves by the Stationary Phase Method

Date: April 30, $1954 \quad$ Place: $39^{\circ} \mathrm{N}, 22^{\circ} \mathrm{E} \quad$ Distance: $10,940 \mathrm{~km} \quad$ Azimuth: $328^{\circ}$

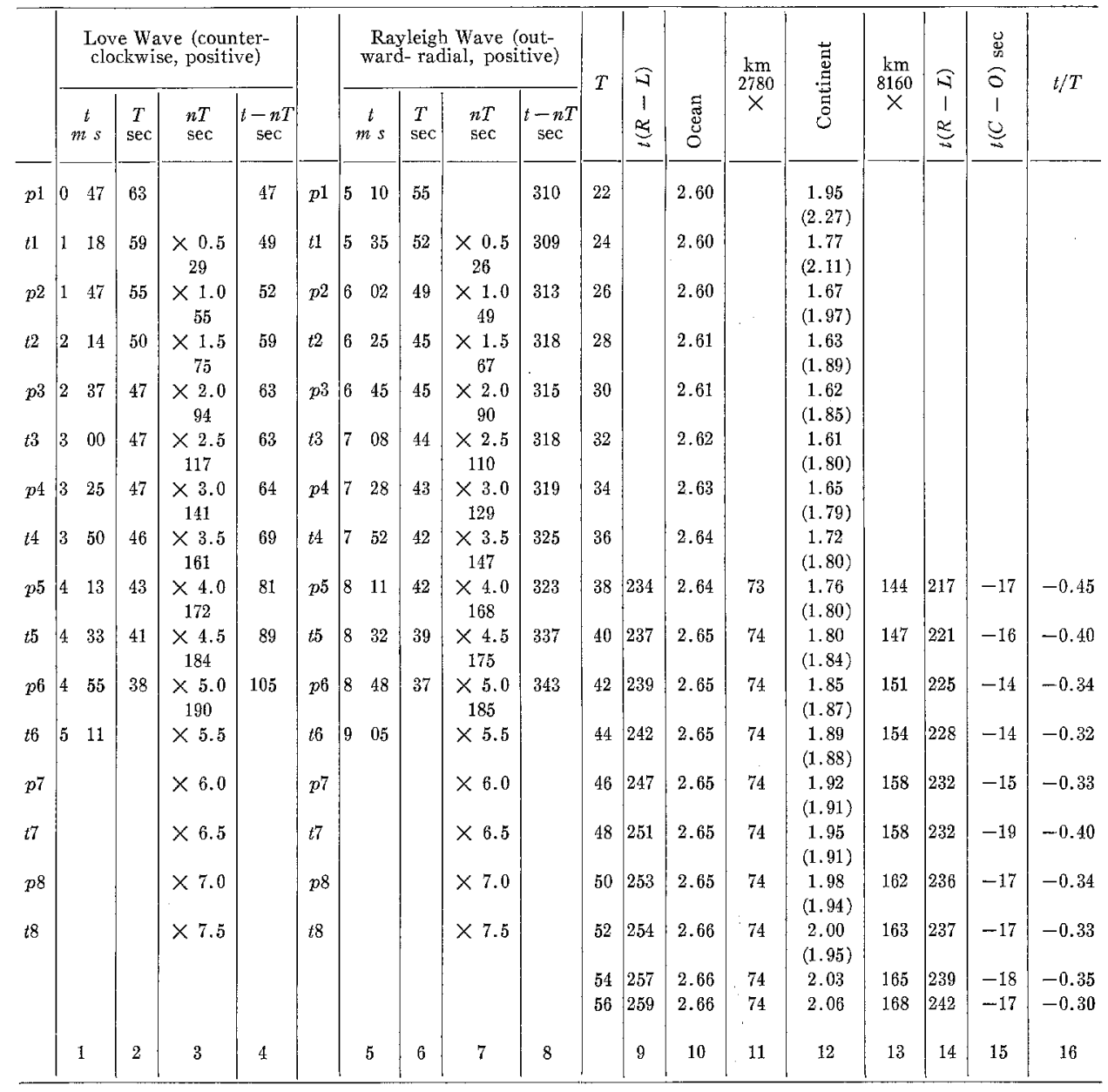

We then compute the same quantity for Rayleigh waves, as is shown in Columns 5 to 8 . We plot this value against the period $2 \pi / \omega_{0}$ for both waves and obtain the difference between them and show it in Column 9 . The numbers in this column may be expressed as

$$
\frac{\left(\phi_{L}{ }^{\prime}(\omega) \pm \frac{\pi}{4}\right)-\left(\phi_{R}{ }^{\prime}(\omega) \pm \frac{\pi}{4}\right)}{\omega}
$$


The choice of sign in this expression is determined according as $d^{2} \phi^{\prime} / d \omega^{2} \gtrless 0$ for each wave. Since $-d \phi^{\prime} / d \omega$ is the group delay time $\Delta / U$, this is equivalent to the condition $d U / d T \lessgtr 0$. In most practical cases dealt with in this paper, we can adopt the same sign for both waves. Then, the numbers in Column 9 may be written as

$$
\frac{\phi_{L}{ }^{\prime}(\omega)-\phi_{R}{ }^{\prime}(\omega)}{\omega}
$$

Columns 10 and 12 show the propagation correction to phase delay time difference between Love and Rayleigh waves for a standard oceanic path and for a standard continental path. The numbers are given in units of second per $100 \mathrm{~km}$. The values for the oceanic path are based on the theoretical model of Sykes et al. (1962), and those for the continental path are based on Press's case 6EG (Press, 1960). The values in brackets in Column 12 are based on Alexander's model (Alexander, 1963). All of these values are corrected for the curvature of the earth. We assume that the structure is oceanic when the ocean depth exceeds 2000 meters, and otherwise continental. The phase delay times for both structures are computed and added as shown in Columns 11, 13 and 14. Column 15 gives the difference between the numbers in Column 14 and 9 , and represents

$$
-\left(\frac{1}{C_{L}(\omega)}-\frac{1}{C_{R}(\omega)}\right) \Delta-\frac{\phi_{L}{ }^{\prime}(\omega)-\phi_{R}{ }^{\prime}(\omega)}{\omega}
$$

This is equal to the source phase difference $\phi_{R}-\phi_{L}$ divided by $\omega$, as defined in equations 3 and 4 . Finally, the last column gives the values of $\phi_{R}-\phi_{L}$ in parts of a circle.

\section{Band-Pass-Filtering and Correlation Method}

The stationary phase method described in the preceding section is difficult to apply to less dispersed waves or to waves buried in noise. The Fourier analysis method may be adequate for these waves. There is, however, a rather serious disadvantage with the Fourier analysis method in that one leaves the time domain and operates entirely in the frequency domain. This is disadvantageous because the seismogram is essentially a mixture of different kinds of waves, each of which has different propagation characteristics; if one works exclusively in the frequency domain, it is difficult for him to see how other waves or noise affect the signal he is concerned with. The apparent accuracy in numbers obtained in the Fourier analysis method is meaningless without an estimate of the error due to the existence of other waves or noise.

For this reason, we prefer to stay in the time domain as long as possible. We shall describe here a program for the IBM 7090 specifically designed for obtaining the phase difference between Love and Rayleigh waves from a given set of three component records. The program consists of three parts: the first part band-pass filters the records; the second part separates Love and Rayleigh waves and tests wave characteristics; the third part performs cross-correlation between Love and Rayleigh waves to obtain the phase difference between them. 
Band-pass-filtering was done by the smoothing of records with a weighting function defined by

$$
\frac{1}{\pi} \int_{\omega_{1}}^{\omega_{2}} \cos \omega t d \omega=\frac{1}{\pi} \frac{\sin \omega_{2} t-\sin \omega_{1} t}{t}
$$

for the two horizontal components, and with one defined as

$$
\frac{1}{\pi} \int_{\omega_{1}}^{\omega_{2}} \sin \omega t d \omega=\frac{1}{\pi} \frac{\cos \omega_{1} t-\cos \omega_{2} t}{t}
$$

for the vertical component. By this operation, the signals outside the frequency range $\omega_{1}$ to $\omega_{2}$ are removed from the records. The phase shift is zero for the horizontal components and $\pi / 2$ advance for the vertical component.

TABLE 2

\begin{tabular}{|c|c|c|c|c|}
\hline Layer Name & Thickness, km & $V_{\mathbf{P}}, \mathrm{km} / \mathrm{sec}$ & $V_{\mathrm{S}}, \mathrm{km} / \mathrm{sec}$ & $\rho, \mathrm{gr} / \mathrm{cm}^{3}$ \\
\hline Sedimentary & 3 & 4.0 & 2.00 & 2.20 \\
\hline Granitic & 23 & 6.2 & 3.70 & 2.78 \\
\hline \multirow{2}{*}{ Intermediate } & $\{8$ & 7.7 & 4.10 & 3.23 \\
\hline & 16 & 7.7 & 4.10 & 3.25 \\
\hline \multirow{3}{*}{ Mantle } & $(15$ & 8.093 & 4.653 & 3.37 \\
\hline & 20 & 7.961 & 4.562 & 3.42 \\
\hline & $\infty$ & 7.835 & 4.480 & 3.43 \\
\hline
\end{tabular}

Layer Parameters of Alexander's Case A1

At the next step, the azimuth to the epicenter from the station is computed, and the transverse and radial motions are synthesized from the smoothed horizontal component records. Then, the correlation coefficient is computed over the Rayleigh wave portion between the radial component and the $\pi / 2$ shifted vertical component. If only the fundamental mode of Rayleigh waves exists in both components, we should get a correlation coefficient of +1 , and if any other waves coexist, the coefficient would be smaller than +1 . The magnitude of this coefficient will indicate the quality of Rayleigh wave data. Allowance was made in the program for the uncertainty in the azimuth of wave approach and also in the magnification ratio of the two horizontal components. We compute the correlation coefficient for possible variations of the above two factors, and choose the ones which give the highest correlation.

Finally, the cross-correlation function $\int L(t) R(t+\tau) d t$ is computed between the transverse component of Love waves $L(t)$ and the radial component of Rayleigh waves $R(t)$, which are filtered and separated in the foregoing steps. An example of the cross-correlation function is shown in figure 7 of Part 2. In order to obtain the phase difference between Love and Rayleigh waves, we first read the time shift $\tau_{p}$ at which the maximum peak of the function appears. Then, we read the period 
at this peak. The phase difference is computed according to the formula

$$
\phi_{R}{ }^{\prime}-\phi_{L}{ }^{\prime}=\frac{\frac{1}{C_{L}(T)}-\frac{1}{C_{R}(T)} \Delta-\tau_{p}}{\frac{T}{2 \pi}}
$$

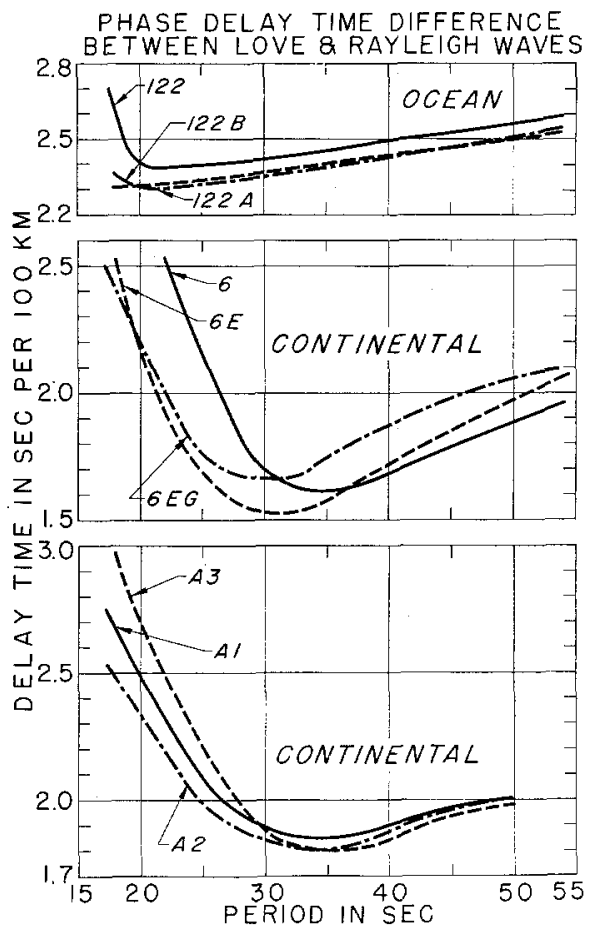

FIG 1. The difference in phase delay time between Love and Rayleigh waves for various models of earth's crustal structure.

Since we usually choose a narrow band width in the filtering, it is not necessary to apply $\pi / 4$ corrections as used in the stationary phase approximation.

The choice of the band width of the filter must be made carefully. If it is too narrow, we lose control of the amplitude variation of the envelope of the crosscorrelation function. In other words, there will be greater interference from other waves if we use a narrow band filter which has a lengthy weighting function. On the other hand, if the band width is too wide, we lose in the accuracy of measuring the period. According to our experience, the appropriate band width seems to be about 0.3 to 0.4 of the center frequency. With this band width, we can not only measure the phase difference between the two waves, but also the group delay time difference between them as will be shown in Part 2 (cf. figure 11 of Part 2).

\section{Phase Velocity Data}

Since the phase velocities of Love and Rayleigh waves in general go together when the crustal structure is varied, it is expected that the difference between them 
would be less sensitive to the crustal structure variation. Figure 1 shows the difference in phase delay time, i.e.,

$$
\frac{1}{C_{R}}-\frac{1}{C_{L}}
$$

in units of second per $100 \mathrm{~km}$, for various models of continental and oceanic structure. They are obtained by the use of "mail order program" for IBM 7090 (Press et al., 1961), which computes the dispersion curves of both Love and Rayleigh waves for a flat layer earth model. Case 6 is a single layer crust overlying a uniform mantle, Case $6 \mathrm{E}$ is a double layer crust overlying the same mantle, and Case $6 \mathrm{EG}$ is the same crust as in $6 \mathrm{E}$ overlying the mantle with a low velocity channel (Press, 1960). We notice that the variation of phase delay time difference with the

TABLE 3

Curvature Correction to the Phase Delay Time of Love and Rayleigh Waves (in see per $100 \mathrm{~km}$ )

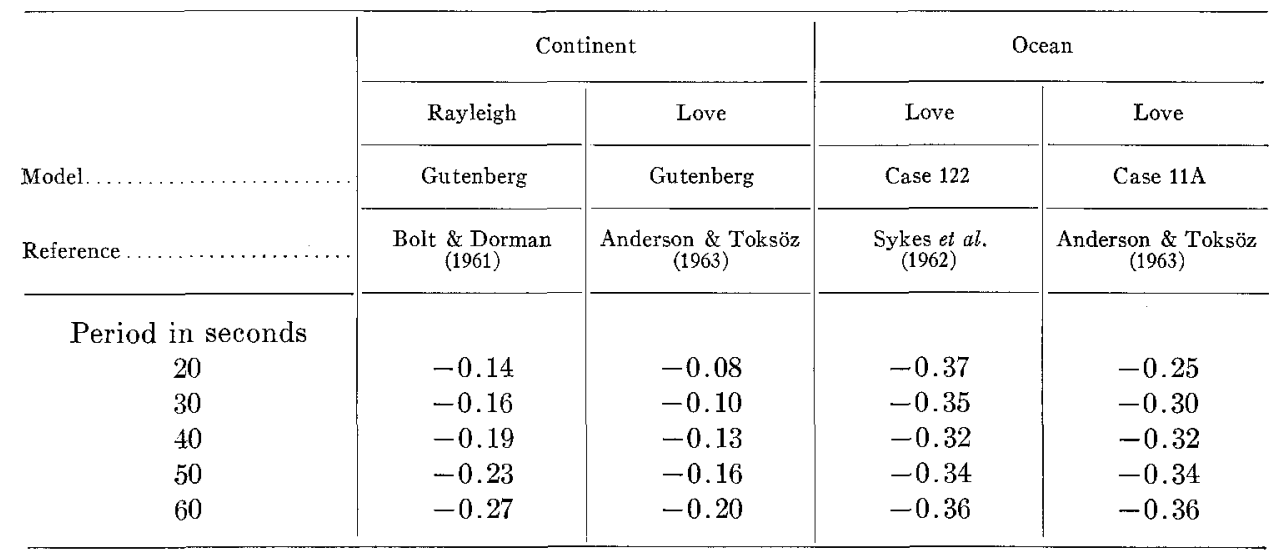

above great change in structure is rather small and less than about 0.2 sec per 100 $\mathrm{km}$ for the period range 28 to $50 \mathrm{sec}$. We also notice that the phase delay time difference shows a minimum value at around $30 \mathrm{sec}$, and varies only slightly with period around this point. This means that a change in total thickness of crust, which merely changes the scale of period axis, will have only a small effect on the phase delay time difference at these periods.

Figure 1 also shows the phase delay time difference for Case A1 of Alexander (1963), for which the layer parameters are given in table 2. This structure explains very well the observed group velocities of fundamental and several higher modes of both Love and Rayleigh waves for the California-Nevada region. We changed this structure in the following two ways. In Case A2, the sedimentary layer was thickened by $1 \mathrm{~km}$ and the granitic layer thinned by the same amount. In Case A3, the granitic layer is thickened by $5 \mathrm{~km}$ and the intermediate layer is thinned by the same amount. As shown in the figure, both changes in the structure have a very small effect (about 0.05 sec per $100 \mathrm{~km}$ ) on the phase delay time difference for periods greater than 28 sec. 
Figure 1 also shows a similar comparison of various oceanic structures. Case 122 was studied by Sykes and others (1962). In Case 122A, the water depth is decreased by $2 \mathrm{~km}$ and the basaltic layer is thickened by the same amount. In Case $122 \mathrm{~B}$, the water depth is decreased by $2 \mathrm{~km}$ and the layer with $V_{p}$ of $4.8 \mathrm{~km} / \mathrm{sec}$ is thickened by the same amount. As shown in the figure, the changes in the phase delay time difference by the above variations in structure are less than 0.1 sec per $100 \mathrm{~km}$. It is remarkable that the phase delay time difference varies very little with period for these oceanic structures.

It has been shown by Sykes et al. (1962) and by Anderson and Toksöz (1962) that the effect of curvature of the earth is more pronounced for oceanic Love waves than for Rayleigh waves in the shorter period range. This is caused by the fact that Love wave energy is more strongly trapped in the low velocity channel in the mantle under oceans. In the case of continental structure, the energy trapped in the channel is small and hence the effect of curvature on the phase velocity is also small. We must, therefore, apply different curvature corrections to our results for flat layer models for continents and oceans. Table 3 lists the correction to the phase delay time of Love and Rayleigh waves obtained by various authors. We used the same curvature correction for oceanic and continental Rayleigh waves.

Finally, we adopted Case 122 as the standard oceanic structure and Case 6EG as the standard continental structure. The values of the phase delay time difference corrected for curvature are given in table 1 . We also listed the values for Case A1, in order to use them for wave paths in the western United States. We accept these values only as a first approximation to the true values for the actual earth and will modify them in the course of the present study when modification is required.

It will be shown in Part 2 that the above standard values are applicable to the wave path from the Mediterranean region to Pasadena for the wave periods 40 to 60 sec. The paths traversing the north American continent from California to Massachusetts will require a slight revision of these values in the periods 28 to 40 sec. A significant modification will be required for the oceanic path from Japan to Pasadena. These results will provide additional information on crustal structure.

\section{A New Model of an Earthquake Source}

It is accepted by the majority of seismologists that an earthquake is a fracture phenomenon which suddenly releases the strain energy stored in a certain volume. However, they still do not agree on the type of simple force system which may best represent this fracture phenomenon. Some believe that the force system equivalent to a shear fracture is a single couple with moment, while others believe that it is a double couple with zero resultant moment. The evidence from $S$ waves should discriminate between these two types of sources. However, as Stauder (1962) states in a review paper, the conclusions of various investigators from $S$ waves to date seem to be as much a function of the observer as of the focal mechanism.

Evidence from surface waves should also be able to discriminate the two types of sources. The difference between them will most strongly show up in Love wave radiation from a strike-slip earthquake. Love waves from the California and Nevada shocks studied by Aki (1960a, 1960b) clearly showed that the hypothesis of a single couple does not explain the observations. The same conclusion has been 
obtained by Ben-Menahem and Toksöz (1963a, 1963b) from very long period waves of several large earthquakes. Additional evidence against the single couple hypothesis will be given in Part 2 of this paper by a comparative study of two Japanese earthquakes with known faulting.

On the other hand, it has been shown that the double couple hypothesis can explain the above observations. It should be, however, noted that these observations are primarily concerned with strike-slip earthquakes. If the double couple is assumed to apply also to a dip-slip earthquake, we get the following results which seem rather unrealistic. First, if the source is a vertical dip-slip fault and is located at the surface of a homogeneous half space, Rayleigh waves will not be generated on the assumption of the double couple (Haskell, 1963). Further, if the double couple is assumed, two reverse dip-slip motions on the faults dipping opposite directions with a dip angle of $45^{\circ}$ will give rise to identical Rayleigh waves at any azimuth. This implies that even though one of the faults shows upward motion on one side and the other shows downward motion on the same side, the double couple hypothesis predicts the same Rayleigh wave motion for them.

Besides, as will be shown in Part 2, more precise comparison of the theory with the observation seem to suggest that the double couple may not always be adequate as the force system representing an earthquake. Therefore, it is desirable to construct a new model of the force system which would give better agreement with observation. In constructing a new model, we tried to be as conservative as possible, and adopted one having an intermediate character between a single couple and a double couple.

Our new model is constructed in the following way. We start with a single couple compatible with a given fault system, and add to it another couple acting perpendicular to the fault strike in the horizontal plane. The additional couple has only a moment around the vertical axis. The magnitude of this moment is taken as the same as the vertical component of the moment of the original couple, and its sense opposite to that of the original. Thus, in the total system, the moment around the vertical axis vanishes, but those moments around the horizontal axes remain as they are in the original couple. This new model is identical to a double couple when the source is a vertical strike-slip fault and is identical to a single couple when the source is a pure dip-slip fault. In general, we may say that the new model looks like a single couple if viewed from the side, and looks like a double couple if viewed from above.

This new model is consistent with the observations by Ben-Menahem and Toksöz (1963a, 1963b) and Aki (1960a, 1960b), because the earthquakes studied by them are due to predominantly strike-slip motion, for which the new model is similar to the double couple.

This new model is also consistent with the assumptions made in some previous studjes of Rayleigh wave radiation from dip-slip earthquakes. Benioff (1963) showed that the record of the Montana earthquake of 1959 at Pasadena agrees well with Lamb's theoretical seismogram based on a single couple source with vertical force direction. Aki (1960c, 1962a, b) also assumed a single couple source, when he interpreted the Rayleigh wave source functions of dip-slip earthquakes in the circumPacific region. 
Further, the new model may not be immediately rejected from the standpoint of the elastic dislocation theory which in general favors the double couple hypothesis (cf. Steketee, 1958, Knopoff and Gilbert, 1960, and Maruyama, 1963). The effect of the free surface on the elastic field due to dip-slip dislocation is not yet fully worked out even for the static case. It seems natural to presume that the free surface acts differently on dip-slip and strike-slip dislocation. Therefore, it is not impossible that the single couple is a better approximation to a dip-slip earthquake than the double couple. Since we emphasize the importance of the free surface, it is implicit that the new model is applicable to shallow earthquakes. It may be that the new model only applies to long period surface waves and not to body waves which probably have too short wave lenoths to know of the existence of the surface when they left the source.

The most important consequence of this new hypothesis would be that we shall have a basis to choose the actual fault plane from the two nodal planes of initial motions by observing surface waves, except for a pure strike-slip earthquake on a vertical fault, for which our new model is identical to a double couple.

We shall hereafter call our new model the modified single couple.

\section{Source Phase and Amplitude of Rayleigh Waves}

The problem of the generation of Rayleigh waves in a homogeneous half space from various types of sources has been studied by many theoretical seismologists since Lamb (1904). Summarizing these results, Aki (1960c, 1962a) obtained the source phase of Rayleigh waves corresponding to simple fault models with vertical fault planes. Recently, Haskell (1963) treated this problem rigorously, and obtained the phase and amplitude radiation pattern for an arbitrary fault system. He studied both single couple and double couple sources, and showed a striking difference in the radiation pattern between the two models. He also demonstrated a very significant effect of the dip of the fault plane on the radiation pattern.

For use in the present study, we have constructed a table of the source phase and amplitude for an arbitrary fault system based on the single couple, double couple and our new model described in the preceding section. In order to match the standard parameters given in the fault plane solutions from body wave data, we specify the dip direction $\varphi_{1}$ of the fault plane, the dip direction $\varphi_{2}$ of the auxiliary plane and the dip angle $\delta_{1}$ of the fault plane. Then, the dip angle $\delta_{2}$ of the auxiliary plane is obtained by the formula,

$$
\tan \delta_{2}=\frac{-1}{\tan \delta_{1} \cos \left(\varphi_{1}-\varphi_{2}\right)}
$$

The slip angle $\sigma$, which is the angle between the strike of the fault and the direction of motion, may be computed by the formula

$$
\cos \sigma=\sin \delta_{2} \sin \left(\varphi_{1}-\varphi_{2}\right)
$$

Then, the components of the force compatible with the above fault motion in the horizontal direction parallel and perpendicular to the fault strike and its vertical 
component may be obtained as

$$
\begin{aligned}
f_{S H} & =F \cos \sigma \\
f_{D H} & =F \sin \sigma \cos \delta_{1} \\
f_{V} & =F \sin \sigma \sin \delta_{1}
\end{aligned}
$$

respectively. These forces correspond to $f_{1}, f_{2}$ and $f_{3}$ in Haskell's (1963) paper. Our sign conventions are such that the forces are positive on the overhanging side of the fault when the motion is left-lateral reverse faulting.

According to the theory (cf. Ben-Menahem, 1961), the horizontal singlet force which is buried at a depth $h$ and varies as $L e^{i \omega t}$ will generate Rayleigh waves with radial displacement of the form

$$
u_{r}=i_{\mu}^{L} K_{\beta} C_{1} \cos \varphi e^{i \omega t}\left\{e^{-h K_{n} d_{1}}-E_{1} e^{-h K_{n} d_{2}}\right\} H_{0}^{(2)}\left(K_{n} r\right)
$$

Here, $K_{n}$ is the wave number of Rayleigh waves, $K_{\beta}$ is the wave number of shear waves, $\varphi$ is the azimuthal angle measured from the direction of force. In the case of the Poisson's solid, for which $\lambda=\mu, d_{1}=0.3933, d_{2}=0.8475$ and $E_{1}=1.7321$.

The radial dispacement corresponding to the source of vertical singlet force is given by the formula

$$
u_{r}=i{ }_{\mu}^{L} K_{\beta} C_{3} e^{i \omega t}\left\{e^{-h K_{n} d_{2}}-E_{1} e^{-h K_{n} d_{1}}\right\} H_{1}^{(2)}\left(K_{n} \gamma\right)
$$

where $C_{3}=1.4678 C_{1}$ for the Poisson solid.

At long distances, we may approximate the Hankel functions in the above equations by sinusoidal functions. Then, we can obtain the phase angle compatible with those from observations as defined in equations 1 and 2.

The Rayleigh wave motion due to an arbitrary force $\left(f_{S H}, f_{D H}, f_{V}\right)$ may be computed as the sum of contributions from each component force. Then, the differentiation of this motion with respect to the direction perpendicular to the fault plane will give the motion corresponding to the source of a single couple. The motion due to a double couple can be obtained by adding to the above motion that due to the single couple corresponding to the fault system in which the fault plane and the auxiliary plane are exchanged. The motion due to the source of the modified single couple can be obtained by adding the contribution from an additional couple which has the force direction perpendicular to the fault strike, and has moment only around the vertical axis with the same magnitude and opposite sense to the corresponding moment of $f_{S H}$ in the original couple.

The computation was made on an IBM 7090 computer. We constructed a table which gives the source phase and amplitude of Rayleigh waves for all possible choices of fault parameters based on the above three simple force systems. It covers the azimuthal angle from 0 to $360^{\circ}$ with $10^{\circ}$ interval, the difference in dip direction 
between the fault and auxiliary plane from $90^{\circ}$ to $270^{\circ}$ with 2 to $5^{\circ}$ interval, the dip angle of the fault plane from $5^{\circ}$ to $90^{\circ}$ with $5^{\circ}$ interval. We computed these values before we saw Haskell's (1963) paper. We found that our results were identical to his for the cases given in his paper.

Our table gives the values for 4 different focal depths, $h=0,0.2 \lambda, 0.4 \lambda$ and $0.6 \lambda, \lambda$ being the wavelength. It may be argued that the assumption of a homogeneous half space is an oversimplification, especially for buried sources. It would be desirable to use the excitation function for an appropriate layered media instead of those for the homogeneous half space. However, there are no serious differences in the excitation functions of a half space and a layered medium, as long as the structure is not as extreme as some of the cases studied by Harkrider, Hales and Press (1963). Therefore, in the present paper we used the values computed on the assumption of a homogeneous half space for Rayleigh waves. For Love waves, however, we shall take into account the presence of layers.

\section{Source Phase and Amplitude of Love Waves}

According to theory (cf. Yanovskaya, 1958, Ben-Menahem, 1961), the transverse displacement of Love waves due to a singlet horizontal force, which varies as $L e^{i \omega t}$, may be expressed as

$$
u_{\varphi}=+\frac{i L}{2 \mu_{1}} \sin \varphi N_{0}(\omega) \cos \left(h \omega \alpha_{1}\right) e^{i \omega t} H_{0}^{(2)}\left(K_{n} r\right)
$$

This expression is derived under the assumption that a homogeneous layer overlies a homogeneous half space, and the source is located within the layer. $N_{0}(\omega)$ is a function of frequency and the layer thickness, and $\varphi$ is the azimuthal angle measured counter clockwise from the force direction. The focal depth appears only in the term $\cos \left(h \omega \alpha_{1}\right)$, where $\alpha_{1}=\sqrt{\left(1 / \beta_{1}^{2}\right)-\left(1 / c^{2}\right)}, \beta_{1}$ being the shear velocity in the layer and $c$ the phase velocity of Love waves.

From reciprocity, it can be shown that the excitation function of Love waves as a function of focal depth will be the same at long distances as the amplitude variation of free Love waves with depth. The latter variation has been computed by various authors (e.g., Anderson and Toksöz, 1962) for several models of the earth's crust and mantle. Their results seem to argue against using a simple formula like $\cos \left(h \omega \alpha_{1}\right)$ in equation 20 . Besides, the amplitude variation depends more strongly on the structure than is the case for Rayleigh waves. However, since there is no change of sign in the amplitude curve of Love waves with increasing depth (which reversal occurs in the horizontal displacement of Rayleigh waves) the source phase of Love waves is much less sensitive to focal depth than is the case for Rayleigh waves.

At long distances, we may rewrite equation 20 in the following form,

$$
u_{\varphi}=-\sin \varphi f(h, \omega) \cos \left(\omega t-K_{n} r-\frac{\pi}{4}\right)
$$

The effect of focal depth $h$ on the phase angle of Love waves appears when we com- 
pute the space derivative of $u_{\varphi}$ to obtain the displacements corresponding to a couple or other type of sources. For instance, the displacement due to a horizontal couple on the fault with the dip angle $\delta$ will be expressed as

$$
\begin{aligned}
& u_{\varphi}^{s}=\left(-\frac{\partial u_{\varphi}}{\partial h} \cos \delta+\frac{\partial u_{\varphi}}{\partial y} \sin \delta\right) d \\
&=d \sin \varphi\left[\frac{\partial f(h, \omega)}{\partial h} \cos \delta \cos \left(\omega t-K_{n} r-\frac{\pi}{4}\right)\right. \\
&\left.\quad-K_{n} \sin \varphi \sin \delta f(h, \omega) \sin \left(\omega t-K_{n} r^{*}-\frac{\pi}{4}\right)\right] \\
&=A \sin \varphi \cos \left(\omega t-K_{n} r-\frac{\pi}{4}+\varphi_{0}\right)
\end{aligned}
$$

where $\varphi_{0}$ is given by the formula

$$
\tan \varphi_{0}=\frac{K_{n} \sin \varphi f(h, \omega)}{\frac{\partial f}{\partial h}} \tan \delta
$$

The expressions for the double couple and the modified single couple may be obtained by superposing the contributions from an additional couple appropriate for each of them.

As shown in equation 23 , the effect of focal depth on the source phase of Love waves is determined by the factor $(\partial f / \partial h) / K_{n} f(h, \omega)$. The amplitude curve of the fundamental mode of Love waves given by Anderson and Toksöz (1963) indicates that this quantity varies from 0.05 to 0.1 for oceans and for 0.1 to 0.5 for continents for the periods and depths we are concerned. We found that the source phase variation in the above range of the parameter $(\partial f / \partial h) / K_{n} f(h, \omega)$ is usually less than 0.03 in parts of a circle, and rarely exceeds 0.1 even near the nodal lines, where the depth effect is the strongest.

The amplitudes of Love waves are also given in our table. They are computed by putting $K_{n} f(h, \omega)$ constant and varying $(\partial f / \partial h) / K_{n} f(h, \omega)$ from 0.1 to 0.4 . The amplitudes are normalized in such a way that the maximum value of Love waves from pure strike-slip motion on a vertical fault takes the same value (0.7054) as the maximum for Rayleigh waves from the same source. In this normalization, the depth for Rayleigh waves is taken as zero and the depth parameter for Love waves is taken as 0.1 . These amplitudes values will, at least, show correctly the variations as a function of the geometry of the fault system.

\section{Application of the Method}

The method described above will be applied in Part 2 to earthquakes in the Mediterranean region, California and Japan. We shall compute the theoretical values of the source phase and amplitude of Love and Rayleigh waves from these 
earthquakes on the assumption of the double couple and the modified single couple, using source information based on geological, geodetic and fault plane studies. Then, we shall compare the theoretical results with the observation, using the technique described in the present paper. The table of the source phase of surface waves for various fault parameters will be given in Part 3 in a concise form.

\section{ACKNOWLEDGEMENTS}

Dr. Frank Press and Dr. Clarence R. Allen kindly read through the manuscript and gave the author valuable suggestions and advice. Discussion with Dr. Ari Ben-Menahem were very helpful to the author. This research was supported by Grant No. AF-AFOSR-25-63 of the Air Force Office of Scientific Research as part of the Advanced Research Projects Agency Project VELA.

\section{REFERENCES}

Aki, K.

1960a. "The use of Love waves for the study of earthquake mechanism," J. Geophys. Res., $65,323-331$.

1960b. "Study of earthquake mechanism by a method of phase equilization applied to Rayleigh and Love waves," J. Geophys. Res., 65, 729-740.

1960c. "Interpretation of source functions of circum-Pacific earthquakes obtained from long period Rayleigh waves," J. Geophys. Res., 65, 2405-2417.

1962a. "Accuracy of the Rayleigh wave method for studying the earthquake mechanism," Bull. Earthquake Res. Inst. Tokyo Univ., 40, 91-105.

1962b. "Revision of some results obtained in the study of the source function of Rayleigh waves," J. Geophys. Res., 67, 3645-3647.

Alexander, S. S.

1963. "Surface wave propagation in the western United States," Doctoral Thesis, California Institute of Technology.

Anderson, D. L. and M. N. Toksöz

1963. "Surface waves on a spherical earth. 1. Upper mantle structure from Love waves," J. Geophys. Res., 68, 3483-3500.

Benioff, $\mathrm{H}$.

1963. "Source wave forms of three earthquakes," report, Contract AF-AFOSR-62-355, California Institute of Technology, Bull. Seism. Soc. Am., 53, 893-904.

Ben-Menahem, A.

1961. "Radiation of seismic surface waves from finite moving sources," Bull. Seism. Soc. Am., 51, 401-435.

Ben-Menahem, A. and M. N. Toksöz

1963a. "Source mechanism from spectra of long period surface waves. 2. The Kamchatka earthquake of Nov. 4, 1952," J. Geophys. Res., 68, 5207-5222.

1963b. "Source mechanism from spectra of long period surface waves. 3 . The Alaska earthquake of July 10, 1958," Bull. Seism. Soc. Am., 53, 905-920.

Bolt, B. A. and J. Dorman

1961. "Phase and group velocities of Rayleigh waves in a spherical gravitating earth," J. Geophys. Res., 66, 2965-2982.

Brune, J. N., J. E. Nafe and J. Oliver

1960. "A simplified method for the analysis and synthesis of dispersed wave trains," $J$. Geophys. Res., 65, 287-304.

Brune, J. N., J. E. Nafe and L. E. Alsop

1961. "The polar phase shift of surface waves on a sphere," Bull. Seism. Soc. Am., 51, $247-258$.

Harkrider, D. G., A. L. Hales and F. Press

1963. "On detecting soft layers in the mantle with Rayleigh waves," Bull. Seism. Soc. Am., 53, 539-548. 
Haskell, N. A.

1963. "Radiation pattern of Rayleigh waves from fault of arbitrary dip and direction of motion in a homogeneous medium," Bull. Seism. Soc. Am., 53, 619-642.

Knopoff, L. and F. Gilbert

1960. "First motion from seismic sources," Bull. Seism. Soc. Am., 50, 117-134.

Lamb, H.

1904. "On the propagation of tremors over the surface of an elastic solid," Phil. Trans. Roy. Soc. London, A, 203, 1-42.

Maruyama, $\mathrm{T}$.

1963. "On the force equivalents of dynamic elastic dislocation with reference to earthquake mechanism," Bull. Earthquake Res. Inst. Tokyo Univ., 41, in press.

Press, F.

1960. "Crustal structure in California-Nevada region," J. Geophys. Res., 65, 1039-1051.

Press, F., D. G. Harkrider and C. A. Seafeldt

1961. "A fast convenient program for computation of surface wave dispersion curves in multi-layered media," Bull. Seism. Soc. Am. 51, 495-502.

Stauder, W.

1962. "The focal mechanism of earthquakes," Advances in Geophysics, Vol. 9, 1-76, Academic Press, New York and London.

Steketee, J. A.

1958. "Some geophysical applications of the elasticity theory of dislocations," Can. $J$. Phys., 36, 1168-1198.

Sykes, L., M. Landisman and Y. Satố

1962. "Mantle shear velocities determined from oceanic Love and Rayleigh wave dispersion," J. Geophys. Res., 67, 5257-5271.

Yanovskaya, T. B.

1958. "On the determination of the dynamic parameters of the focus and hypocentre of an earthquake from records of surface waves," Izv. Akad. Nauk USSR, Ser. Geofiz., 289-301.

Seismological Laboratory

California Institute of Teghnology

Pasadena, California

Division of the Geological Sciences Contribution No. 1212

Manuscript received November 21, 1963. 